\title{
Efficacy of Combination Therapy with MET and VEGF Inhibitors for MET-overexpressing Glioblastoma
}

\author{
TAKESHI OKUDA ${ }^{1}$, TAKAYUKI TASAKI ${ }^{1}$, SUSUMU NAKATA ${ }^{2}$, KIMIHIRO YAMASHITA $^{3}$, \\ HIROMASA YOSHIOKA ${ }^{1}$, SHUICHI IZUMOTO ${ }^{1}$, AMAMI KATO ${ }^{1}$ and MITSUGU FUJITA ${ }^{4}$ \\ Departments of ${ }^{1}$ Neurosurgery and ${ }^{4}$ Microbiology, Faculty of Medicine, Kindai University, Osaka, Japan; \\ ${ }^{2}$ Department of Clinical Oncology, Kyoto Pharmaceutical University, Kyoto, Japan; \\ ${ }^{3}$ Department of Surgery, Division of Gastrointestinal Surgery, \\ Kobe University Graduate School of Medicine, Kobe, Japan
}

\begin{abstract}
Background: Glioblastoma multiforme (GBM) is a malignant brain tumor with an extremely poor prognosis. GBM tissues frequently express mesenchymal-epithelial transition factor (MET), which induces cell division, growth and migration. In addition, angiogenesis is a significant feature of GBM, attributable to the overexpression of vascular endothelial growth factor (VEGF). Although the $V E G F$ inhibitor bevacizumab was recently highlighted as the second-line drug for GBM treatment, GBMs often recur even with bevacizumab therapy. Based on these findings, we hypothesized that inhibition of both MET and VEGF would exhibit a synergistic effect on MET-overexpressing GBM. Materials and Methods: As we observed MET expression at high levels in some patients with GBM, we designed GL261 murine glioma-based experiments. GL261 cells were transfected with siRNAs specific for MET and VEGF in vitro, and the cell growth ratios were evaluated. Simultaneously, transfected GL261 cells were transplanted into the brain of C57BL/6 mice, and their survival was monitored. Results: GBM tissues frequently overexpressed MET protein at high levels compared with lower-grade gliomas. These GBMs at first responded to bevacizumab, but often eventually recurred. When GL261 cells were co-transfected with both MET-specific siRNA and VEGF-specific siRNA, the in vitro tumor cell growth significantly decelerated compared to
\end{abstract}

Correspondence to: Takeshi Okuda, MD, Ph.D., Department of Neurosurgery, Faculty of Medicine, Kindai University, Osaka, Japan. Tel: +81 723660221 ext. 3547, Fax: +81 723656975, e-mail: okuda@med.kindai.ac.jp and Mitsugu Fujita, MD, Ph.D., Department of Microbiology, Faculty of Medicine, Kindai University, Osaka, Japan. Tel: +81723660221 ext. 5456, Fax: +81 723660206, e-mail: mfujita47@gmail.com

Key Words: MET, VEGF, glioblastoma. single siRNA transfection. Consistently, when mice were transplanted with co-transfected GL261 cells, their survival was significantly prolonged compared to those given cells transfected with single siRNA. Conclusion: The current data indicate that the inhibition of both MET and VEGF exhibits efficient therapeutic effects of GBM-bearing hosts.

Glioblastoma multiforme (GBM) is a malignant brain tumor that is classified as grade IV by the World Health Organization. The treatment strategy for GBM comprises multimodalities that include surgical removal, radiotherapy, and chemotherapy based on the alkylating agent temozolomide (1). Nevertheless, complete recovery is technically unachievable because of the invasive nature of GBM. The presence of the blood-brain barrier in the brain parenchyma also reduces the sensitivity of GBM to chemoradiotherapy. These characteristics of GBM result in an extremely poor prognosis compared to that of malignant tumors in other organs, with median survival periods of 1215 months $(1,2)$.

Angiogenesis is a significant feature of GBM, and is attributable to the overexpression of vascular endothelial growth factor (VEGF) at tumor sites (3). A VEGF inhibitor, bevacizumab, was recently highlighted as the second-line drug for GBM treatment. Bevacizumab-based treatment for recurrent GBM achieves rapid improvement of clinical signs, including the reduction of cerebral edema and the contraction of the area of contrast on imaging (4). Therapeutic outcome is also favorable in those with recurrent GBM, with a 6month progression-free survival rate of $20-50 \%$ and a median survival of 7-12 months (5). However, when patients with newly diagnosed GBM are treated with bevacizumab and temozolomide simultaneously, the therapeutic outcome becomes unfavorable $(6,7)$. That is, although treatment with bevacizumab initially reduces the tumor size, the tumors eventually enlarge (8-10). 
Mesenchymal-epithelial transition factor (MET), also known as hepatocyte growth factor receptor, functions as a receptor for hepatocyte growth factor (HGF). It is a membrane receptor tyrosine kinase and is expressed in epithelial cells, liver, pancreas, prostate, kidney, and bone marrow (11). The MET-HGF signal induces cell division, growth, and cell migration (11). MET is also a known protooncogene for a variety of malignant tumors, including small cell lung cancer, breast carcinoma, prostatic carcinoma, hepatocellular carcinoma, as well as GBM (12). We and others have reported that MET-overexpressing GBMs are resistant to chemotherapy and confer a poor prognosis (13) and that MET can be a therapeutic target for GBM (14).

Nevertheless, from a viewpoint of targeting MET and VEGF together, administration of VEGF inhibitors is known to enhance MET gene expression in GBM (15). Based on the above findings, we hypothesized that inhibition of both MET and VEGF would exhibit a synergistic effect on METoverexpressing GBM (16).

\section{Materials and Methods}

Patients and immunohistochemistry (IHC). GBM patient-relevant data presentation was approved by the Institutional Review Board of Kindai University (Approval Number: 26-079) (13). The procedure of IHC was described previously (13). Briefly, surgical specimens were fixed with formalin and embedded in paraffin. The tissue samples were then cut into slices of $7 \mu \mathrm{m}$ thick. The tissue sections were then heated three times for $5 \mathrm{~min}$ in target-retrieval solution (Aligent Technologies, Santa Clara, CA, USA) and stained with monoclonal antibody to total MET (Clone D1C2; Cell Signaling Technology, Danvers, MA, USA) or normal rabbit IgG antibody followed by biotin-labeled goat antirabbit IgG antibody and Vectastain staining kit (Vector Laboratories, Burlingame, CA, USA). After enzymatic development, the sections were counterstained with Gill's hematoxylin. Two pathologists independently evaluated the expression levels of MET.

Reagents and cell culture. The procedure used here has been described previously $(17,18)$. Briefly, RPMI1640, fetal bovine serum (FBS), L-glutamine, sodium pyruvate, $\beta$-mercaptoethanol, nonessential amino acids and antibiotics were obtained from SigmaAldrich (Grand Island, NY, USA). GL261 mouse glioma cell line was kindly provided from Aichi Cancer Center Research Institute, where GL261 cells were from C57BL/6 mouse background (17-19). The cells were maintained in mouse complete medium (RPMI-1640 supplemented with $10 \%$ heat-inactivated FBS, 100 units $/ \mathrm{ml}$ penicillin, $100 \mathrm{mg} / \mathrm{ml}$ streptomycin, and $10 \mu \mathrm{M}$ L-glutamine) in a humidified incubator in $5 \% \mathrm{CO}_{2}$ at $37^{\circ} \mathrm{C}$.

Small interfering RNA. The procedure used here has been described previously (20). Briefly, GL261 cells were transfected with siRNA against MET, VEGF, or control siRNA (Santa Cruz Biotechnology, Santa Cruz, CA, USA) using Lipofectamine RNAi MAX (Life Technologies, Waltham, MA, USA) per the manufacturer's instructions with minor modifications. In some experiments, cell viability was assessed after $72 \mathrm{~h}$ of treatment using the Cell Counting Kit 8 (CCK8; Wako, Tokyo, Japan) per the manufacturer's instructions.
GBM-bearing mouse model. The procedure has been described previously $(17,18,20)$. Briefly, wild-type C57BL/6 mice were obtained from CLEA (Tokyo, Japan) and maintained under specific pathogen-free conditions at the Animal Research Center of Kindai University Faculty of Medicine. On day 0, using a Hamilton syringe (Hamilton Company, NV, USA), $1 \times 10^{5}$ GL261 cells in $2 \mu$ PBS were stereotactically injected through an entry site at the bregma, $3 \mathrm{~mm}$ to the right of sagittal suture, and $4 \mathrm{~mm}$ below the surface of the skull of anesthetized mice by using a stereotactic frame (Stolting, Wood Dale, IL, USA). Symptom-free survival was monitored. All procedures were approved by the Institutional Animal Care and Use Committee.

Statistical analyses. The procedure has been described previously (13, 21). Briefly, one-way analysis of variance with Holm's post-hoc test was used for multiple group comparison, and log-rank test was performed to analyze survival. All data were analyzed using $\mathrm{R}$ Environment (R Development Core Team, Vienna, Austria) with EZR plugin (22). A value of $p<0.05$ was considered statistically significant.

\section{Results}

MET-overexpressing GBMs frequently recur after bevacizumab-based VEGF inhibition. Consistent with previous reports (8-10), we also recorded GBM recurrence in patients after treatment with bevacizumab. As we previously showed (13), GBM tissues frequently overexpressed MET protein at high levels compared to lower-grade gliomas (Figure 1A). These GBMs responded to bevacizumab at first (Figure 1B), but most of the tumors recurred eventually. These observations suggest that MET and VEGF are functionally associated with each other and that inhibition of MET and VEGF would be beneficial in controlling the growth of GBM cells.

Inhibition of MET and VEGF synergistically inhibits GBM cell growth in vitro. Cultured GL261 glioma cells express high levels of MET (23) and VEGF (24). In order to confirm the impact of MET inhibition on tumor cell growth, we used siRNA specific for MET in this study (Figure 2). In addition, to address whether VEGF inhibition would enhance the therapeutic impact of MET inhibition, we simultaneously used siRNA specific for VEGF. MET-specific siRNA significantly inhibited the growth of GL261 cells compared with the control siRNA $(p<0.001)$. Similarly, VEGF-specific siRNA also significantly inhibited tumor growth $(p<0.001)$. When the cells were co-transfected with both MET-specific and VEGF-specific siRNA, the tumor growth significantly decelerated compared with single siRNA transfection $(p=0.00432)$. These data indicate that inhibition of both MET and VEGF synergistically inhibits the growth of GL261 murine glioma cells.

Inhibition of MET and VEGF synergistically inhibits GBM cell growth in vivo. These results led us to hypothesize that MET/VEGF-targeting therapy would also exhibit therapeutic efficacy for GBM-bearing hosts in vivo. In order to address 
A

MET-negative GBM

MET-positive GBM

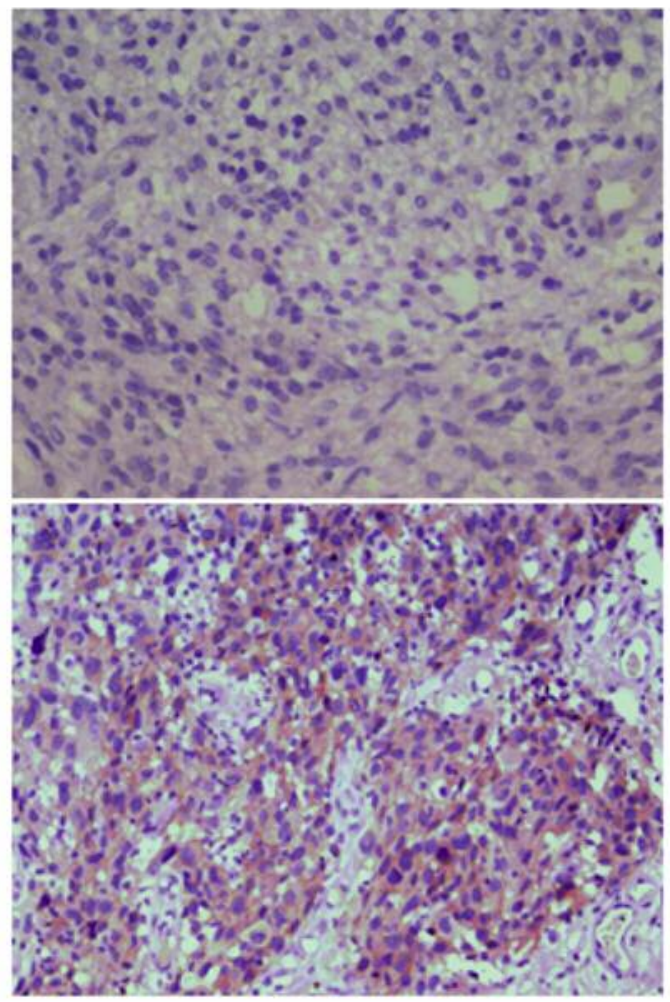

B

Contrast-enhanced

T2-weighted

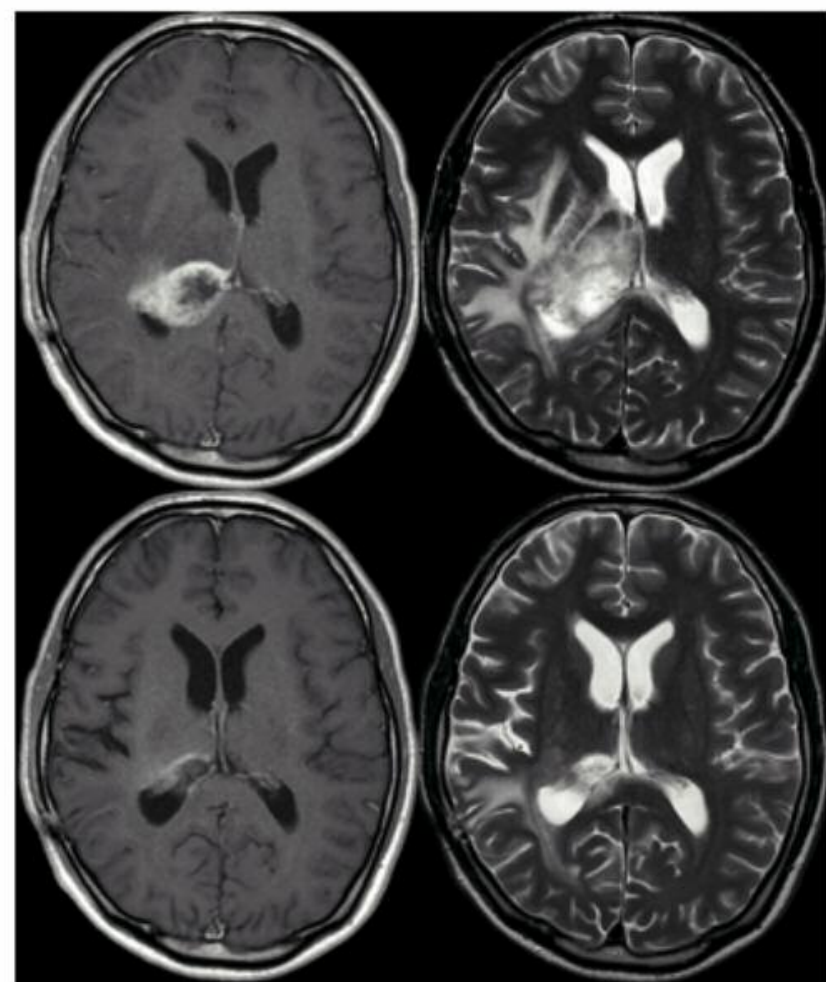

Figure 1. Immunohistochemistry of mesenchymal-epithelial transition factor (MET) expressed in recurrent glioblastoma multiforme (GBM). A, representative images of MET-negative and MET-positive GBM cases. Original magnification: 200-fold. B, Magnetic resonance images of the patient with MET-positive GBM. The tumor responded to bevacizumab treatment. 


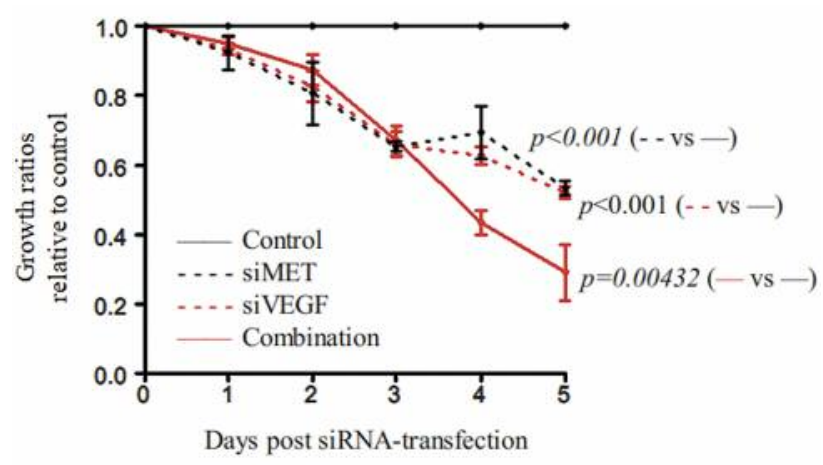

Figure 2. Inhibition of mesenchymal-epithelial transition factor (MET) and vascular endothelial growth factor (VEGF) synergistically inhibits GL261 murine glioma cell growth in vitro. GL261 cells were transfected with siRNAs specific for MET or VEGF or both. Cell growth ratios were calculated relative to growth of the control groups. p-Values were based on Holm's post-hoc test and are shown for comparison with the Control. $p<0.05$ was considered statistically significant.

this question, we established intracerebral GBM mouse models using GL261 cells that were treated with METspecific siRNA with/without VEGF-specific siRNA prior to cell transplantation and then monitored the survival of the GBM-bearing mice (Figure 3). Consistent with the in vitro data, the mice with GL261 treated with MET-specific siRNA exhibited prolonged survival compared with those with untreated GL261 cells $(p=0.0281)$. The mice with GL261 treated with VEGF-specific siRNA also exhibited prolonged survival, which was almost equivalent to that of those with MET-specific siRNA treatment $(p=0.0325)$. When the cells were co-transfected with both MET-specific and VEGFspecific siRNA, the mice with co-transfected GL261 cells survived significantly longer compared with those transplanted with single siRNA transfected GL261 cells $(p<0.001)$. Taken together, these data indicate that the inhibition of both MET and VEGF exhibits more efficient therapeutic effects on GBM-bearing mice.

\section{Discussion}

Our data demonstrated that the combination therapy with both MET and VEGF inhibition promoted favorable therapeutic effects on MET-overexpressing GBMs. MET expression in GBM is associated with tumor resistance to radiation and chemotherapy. VEGF inhibitors suppress angiogenesis at tumor sites. As a result, tumor cells are exposed to a hypoxic microenvironment. This hypoxic microenvironment further enhances $M E T$ gene expression and promotes tumor growth (11). It is therefore important to inhibit both MET and VEGF in order to control the growth of GBM.

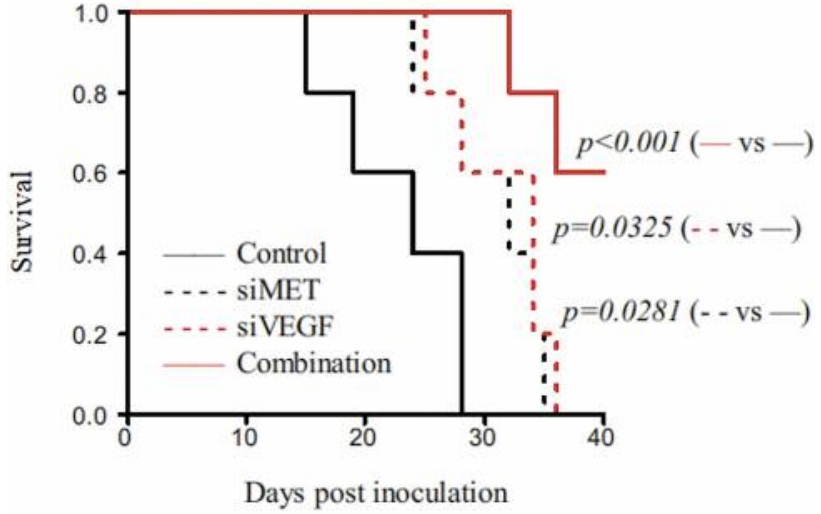

Figure 3. Inhibition of mesenchymal-epithelial transition factor (MET) and vascular endothelial growth factor (VEGF) synergistically inhibits glioblastoma multiforme (GBM) cell growth in vivo. GL261 murine glioma cells were transfected with siRNAs specific for MET or VEGF, or both and the cells were transplanted into the brain of C57BL/6 mice. Survival of the GBM-bearing mice was monitored.p-Values were based on log-rank test and are shown for comparison with the Control. $p<0.05$ was considered statistically significant.

We used GL261 murine glioma cell line (Figure 2). GL261 has often been used for C57BL/6-based animal models for preclinical studies $(17-19,23,24)$. Most importantly, this system allows us to use immunocompetent animals and therefore to evaluate immune reactions in GBMbearing hosts. Moreover, this cell line is easy to maintain and handle. This cell line is known to expresses GP100 as a glioma-associated antigen. In parallel with the direct effects of MET/VEGF inhibition on GBM growth, we address the immunological aspects using this system in response to MET/VEGF inhibition.

We observed favorable therapeutic effects of the combined therapy on GBM in C57BL/6 wild-type mice (Figure 3). In this regard, we recently developed a new animal model of GBM in which spontaneous GBM formation can be induced (25-27). This system mimics human GBM well but the disadvantage of the new system is that tumor-inducible rate is still unstable. In parallel with this new system, we used human-derived GBM cells in immune-deficient mice (13, 20 ). With this system, however, we are unable to address the immunological responses in the GBM microenvironment. Based on these findings, we chose to use the GL261-based cell transplantation model in this study to gain stability of tumor formation in the immunocompetent animals.

Previous studies have shown that bevacizumab exerts short-term effects on glioblastoma (8-10). In this regard, our data and others (28) suggest that this phenomenon is attributable to MET overexpression mediated by VEGF inhibition. We will continue to investigate the effects of 
combination therapy with VEGF inhibitor and MET inhibitor for GBM as has been conducted in melanoma (16).

\section{Acknowledgements}

This study was supported by the following grants: Grant-in-Aid for Scientific Research (C) (No. 15K10347 for TO and No. 15K10348 for MF) from the Ministry of Education, Culture, Sports, Science and Technology (MEXT). The Authors have no conflict of interest to disclose in regard to this study.

\section{References}

1 Stupp R, Mason WP, van den Bent MJ, Weller M, Fisher B, Taphoorn MJ, Belanger K, Brandes AA, Marosi C, Bogdahn U, Curschmann J, Janzer RC, Ludwin SK, Gorlia T, Allgeier A, Lacombe D, Cairncross JG, Eisenhauer E and Mirimanoff RO: Radiotherapy plus concomitant and adjuvant temozolomide for glioblastoma. N Engl J Med 352: 987-996, 2005.

2 Minniti G, Muni R, Lanzetta G, Marchetti P and Enrici RM: Chemotherapy for glioblastoma: current treatment and future perspectives for cytotoxic and targeted agents. Anticancer Res 29: 5171-5184, 2009.

3 Reifenberger G, Wirsching HG, Knobbe-Thomsen CB and Weller M: Advances in the molecular genetics of gliomas implications for classification and therapy. Nat Rev Clin Oncol, 2016. doi: 10.1038/nrclinonc.2016.204 [Epub ahead of print]

4 Campos B, Olsen LR, Urup T and Poulsen HS: A comprehensive profile of recurrent glioblastoma. Oncogene 35: 5819-5825, 2016.

5 Narita Y: Drug review: Safety and efficacy of bevacizumab for glioblastoma and other brain tumors. Jpn J Clin Oncol 43: 587595, 2013.

6 Chinot OL, Wick W, Mason W, Henriksson R, Saran F, Nishikawa R, Carpentier AF, Hoang-Xuan K, Kavan P, Cernea $\mathrm{D}$, Brandes AA, Hilton $\mathrm{M}$, Abrey $\mathrm{L}$ and Cloughesy $\mathrm{T}$ : Bevacizumab plus radiotherapy-temozolomide for newly diagnosed glioblastoma. N Engl J Med 370: 709-722, 2014.

7 Gilbert MR, Dignam JJ, Armstrong TS, Wefel JS, Blumenthal DT, Vogelbaum MA, Colman H, Chakravarti A, Pugh S, Won M, Jeraj R, Brown PD, Jaeckle KA, Schiff D, Stieber VW, Brachman DG, Werner-Wasik M, Tremont-Lukats IW, Sulman EP, Aldape KD, Curran WJ Jr. and Mehta MP: A randomized trial of bevacizumab for newly diagnosed glioblastoma. N Engl J Med 370: 699-708, 2014.

8 Iwamoto FM, Abrey LE, Beal K, Gutin PH, Rosenblum MK, Reuter VE, DeAngelis LM and Lassman AB: Patterns of relapse and prognosis after bevacizumab failure in recurrent glioblastoma. Neurology 73: 1200-1206, 2009.

9 Norden AD, Young GS, Setayesh K, Muzikansky A, Klufas R, Ross GL, Ciampa AS, Ebbeling LG, Levy B, Drappatz J, Kesari $\mathrm{S}$ and Wen PY: Bevacizumab for recurrent malignant gliomas: efficacy, toxicity, and patterns of recurrence. Neurology 70: 779787, 2008.

10 Quant EC, Norden AD, Drappatz J, Muzikansky A, Doherty L, Lafrankie D, Ciampa A, Kesari S and Wen PY: Role of a second chemotherapy in recurrent malignant glioma patients who progress on bevacizumab. Neuro Oncol 11: 550-555, 2009.

11 Organ SL and Tsao MS: An overview of the c-MET signaling pathway. Ther Adv Med Oncol 3: S7-S19, 2011.
12 Boccaccio C and Comoglio PM: Invasive growth: a MET-driven genetic programme for cancer and stem cells. Nat Rev Cancer 6: 637-645, 2006.

13 Tasaki T, Fujita M, Okuda T, Yoneshige A, Nakata S, Yamashita $\mathrm{K}$, Yoshioka $\mathrm{H}$, Izumoto $\mathrm{S}$ and Kato $\mathrm{A}$ : MET expressed in glioma stem cells is a potent therapeutic target for glioblastoma multiforme. Anticancer Res 36: 3571-3577, 2016.

14 Huse JT and Holland EC: Targeting brain cancer: advances in the molecular pathology of malignant glioma and medulloblastoma. Nat Rev Cancer 10: 319-331, 2010.

15 Piao Y, Park SY, Henry V, Smith BD, Tiao N, Flynn DL and de Groot JF: Novel MET/TIE2/VEGFR2 inhibitor altiratinib inhibits tumor growth and invasiveness in bevacizumab-resistant glioblastoma mouse models. Neuro Oncol 18: 1230-1241, 2016.

16 Daud A, Kluger HM, Kurzrock R, Schimmoller F, Weitzman AL, Samuel TA, Moussa AH, Gordon MS and Shapiro GI: Phase II randomised discontinuation trial of the MET/VEGF receptor inhibitor cabozantinib in metastatic melanoma. Br J Cancer 116: 432-440, 2017.

17 Fujita M, Zhu X, Sasaki K, Ueda R, Low KL, Pollack IF and Okada H: Inhibition of STAT3 promotes the efficacy of adoptive transfer therapy using type-1 CTLs by modulation of the immunological microenvironment in a murine intracranial glioma. J Immunol 180: 2089-2098, 2008.

18 Fujita M, Zhu X, Ueda R, Sasaki K, Kohanbash G, Kastenhuber ER, McDonald HA, Gibson GA, Watkins SC, Muthuswamy R, Kalinski $\mathrm{P}$ and Okada $\mathrm{H}$ : Effective immunotherapy against murine gliomas using type 1 polarizing dendritic cellssignificant roles of CXCL10. Cancer Res 69: 1587-1595, 2009.

19 Prins RM, Odesa SK and Liau LM: Immunotherapeutic targeting of shared melanoma-associated antigens in a murine glioma model. Cancer Res 63: 8487-8491, 2003.

20 Yoshioka H, Okuda T, Fujita M, Inoue T, Tasaki T, Izumoto S and Kato A: Inhibition of ABCG2 enhances chemo-sensitivity of murine glioma stem cell-like cells to temozolomide and reduces spheroid-forming capability. Acta Med Kinki Univ 39: 105-113, 2014.

21 Otsubo D, Yamashita K, Fujita M, Nishi M, Kimura Y, Hasegawa H, Suzuki S and Kakeji Y: Early-phase treatment by low-dose 5-fluorouracil or primary tumor resection inhibits MDSC-mediated lung metastasis formation. Anticancer Res 35: 4425-4431, 2015.

22 Kanda Y: Investigation of the freely available easy-to-use software 'EZR' for medical statistics. Bone Marrow Transplant 48: 452-458, 2013.

23 Towner RA, Ihnat M, Saunders D, Bastian A, Smith N, Pavana RK and Gangjee A: A new anti-glioma therapy, AG119: preclinical assessment in a mouse GL261 glioma model. BMC Cancer 15: 522, 2015.

24 Zagzag D, Esencay M, Mendez O, Yee H, Smirnova I, Huang Y, Chiriboga L, Lukyanov E, Liu M and Newcomb EW: Hypoxiaand vascular endothelial growth factor-induced stromal cellderived factor-1alpha/CXCR4 expression in glioblastomas: one plausible explanation of Scherer's structures. Am J Pathol 173: 545-560, 2008.

25 Fujita M, Scheurer ME, Decker SA, McDonald HA, Kohanbash G, Kastenhuber ER, Kato H, Bondy ML, Ohlfest JR and Okada $\mathrm{H}$ : Role of type 1 IFNs in antiglioma immunosurveillance-using mouse studies to guide examination of novel prognostic markers in humans. Clin Cancer Res 16: 3409-3419, 2010. 
26 Fujita M, Kohanbash G, Fellows-Mayle W, Hamilton RL, Komohara Y, Decker SA, Ohlfest JR and Okada H: COX-2 blockade suppresses gliomagenesis by inhibiting myeloidderived suppressor cells. Cancer Res 71: 2664-2674, 2011.

27 Kohanbash G, McKaveney K, Sakaki M, Ueda R, Mintz AH, Amankulor N, Fujita M, Ohlfest JR and Okada H: GM-CSF promotes the immunosuppressive activity of glioma-infiltrating myeloid cells through interleukin-4 receptor-alpha. Cancer Res 73: 6413-6423, 2013.
28 Goel HL and Mercurio AM: VEGF targets the tumour cell. Nat Rev Cancer 13: 871-882, 2013.

Received May 2, 2017

Revised May 18, 2017

Accepted May 19, 2017 